\title{
Design of a Smart Garment for Cycling
}

\author{
André Paiva ${ }^{2[0000-0002-5564-2081]}$, Daniel Vieira ${ }^{1}$, Joana Cunha ${ }^{20000-0001-5063-1124]}$, Hélder \\ Carvalho $^{20000-0003-0472-1107]}$ and Bernardo Providência ${ }^{1}$ \\ ${ }^{1}$ University of Minho, Landscapes, Heritage and Territory Laboratory, Guimarães, Portugal \\ ${ }^{2}$ University of Minho, Centre for Textile Science and Technology, Guimarães, Portugal \\ dmpaiva.s@gmail.com, mail@danielvieira.pt, \\ jcunha@| hcarvalho@det.uminho.ptझ", \\ providenciaearquitectura.uminho.pt
}

\begin{abstract}
Given the premise that cycling encourages a healthy lifestyle and promotes wellbeing, this paper describes the design process for the development of a cycling garment, with embedded electrodes for heart rate measurement to further widen the possibilities of health and performance monitoring, in a practical and unobtrusive way. Electrodes were produced and tested, using different textile materials and techniques. Signal measurement is based on the BBB Y8YBH20 heart rate monitor and performance tests were done using the Polar Beat mobile app. Finally, a prototype of the garment is presented.
\end{abstract}

Keywords: e-textile; wearables; smart textiles; cycling wear; design.

\section{Introduction}

Information technologies (ICT), portable technologies and smart textiles are changing the way we think and design new concepts and paradigms for the area of sports and well-being. With the advent of new types of textiles, such as e-textiles (i.e.: for heart or muscle activity monitoring), the textile becomes a platform to integrate new technologies and increment functionalities. By adding computational technologies, the textile becomes "smart", providing the user information in a ubiquitous way, promoting a new form of interaction with the textile.

This paper describes the design process of a skinsuit for road cycling, triathlon, track cycling, mountain cycling and cyclocross, where e-textile technology for heart rate analysis was explored. For this, we had to understand: (i) the purpose of the concept; (ii) the used technology; (iii) the context in which the product is used. 


\section{State of the Art}

\subsection{Exploring the Technology: Textile Electrodes and Sensors}

The study of textile sensors for vital signs and biometrics has been driven by the idea of building remote monitoring systems that can be continuously connected to the body without compromising comfort, just like clothing, adding new functionalities.

In electrocardiography (ECG), surface electrodes are used, the same type as used in surface EMG and EEG. Several textile materials and techniques have been used in order to produce textile electrodes. Some examples include knitting [1, 2], weaving [3, 4], and embroidery [5], using conductive yarns; printing with conductive ink [6], coating with conductive rubber [7].

Yao [8] have developed an X-band with three conventional silicon electrodes for continuous monitoring of cardiac activity. Zięba et al. [9] created a sleeveless seamless shirt that includes a knitted stretch sensor to measure breathing rate. Manero et al. [10] have presented a prototype of running legging with three pairs of embroidered electrodes (and circuits) for continuous monitoring of muscular activity in the quadriceps. Ma et al. [11] proposed a shirt with textile electrodes for ECG and EMG and a textile breathing sensor, as well as conventional accelerometers and temperature sensor. Frydrysiak and Tesiorowski [12] proposed a remote monitoring system, which includes textiles sensors attached to a shirt for measurement of heart rate, breathing rate and temperature.

A few commercial brands have been investing in smart clothing, mainly for the sports market. Hexoskin [13] has introduced in the market a shirt that combines textile and conventional sensors to provide data about heart rate and variability, breathing rate, oximetry and activity parameters. AiQ Smart Clothing developed Bioman, a shirt that has embedded sensors to monitor heart rate, breathing rate and skin temperature [14]. Athos ${ }^{\circledR}$ gave form to a compression shirt and shorts for sports that makes use of printed sensors with conductive rubber to provide information about heart rate, respiration and muscle activity [15]. Myontec produced cycling shorts with textile electrodes sewn to the shorts to detect electrical signals from quadriceps and hamstrings muscles [16].

So far, a truly invisible integration of technology does not yet seem to be possible, or at least, none is known to be in the market. Nonetheless, all visible technology is designed in order to look pleasantly (and even unnoticed), as if it is part of the garment and not just some strange object.

\section{$3 \quad$ Materials and Methods}

The project follows a design process suggested by the authors that requires the understanding: (i) the purpose of the concept; (ii) the used technology; (iii) the context in which the product is used. Various materials have been explored to make textile electrodes, in order to find a suitable material and method to integrate ECG electrodes in the garment. Performance tests were done using the Polar Beat mobile app. To make a prototype of the suit (size $S$ ), patterns were designed and cut, first for a partial prototype 
and later for a full prototype of the garment. After each fitting, a new prototype was done to correct the size measurements.

\subsection{Design Process}

A product was designed that responds to contemporary standards for cycling apparel and technology, combined in a fashionable way. The designed product is a skinsuit (Fig. 1), that integrates ECG electrodes and a heart monitoring device to continuously monitor heart rate, integrated in a way that it is almost invisible, therefore without compromising aesthetics. A polyamide/elastane jersey fabric was chosen for the cycling garment, which is smooth and provides some compression, keeping the electrodes in contact with the body.

The garment was designed with raglan sleeves, but the position of the ECG device and connections' shape made it impossible, because of the scye seams. It was then decided to use a conventional sleeve, so the shoulder seam could be removed. The electrodes would be put near the frontal center, but given the changes in body shape, the electrodes would not have a good electrical contact. Therefore, it was settled that they would be moved closer to the side, below the chest, where the body shape is more uniform. The heart rate monitoring device (BBB Bluepulse Heart Rate Sensor) was put in the back, near the collar, so it would not interfere with movements.

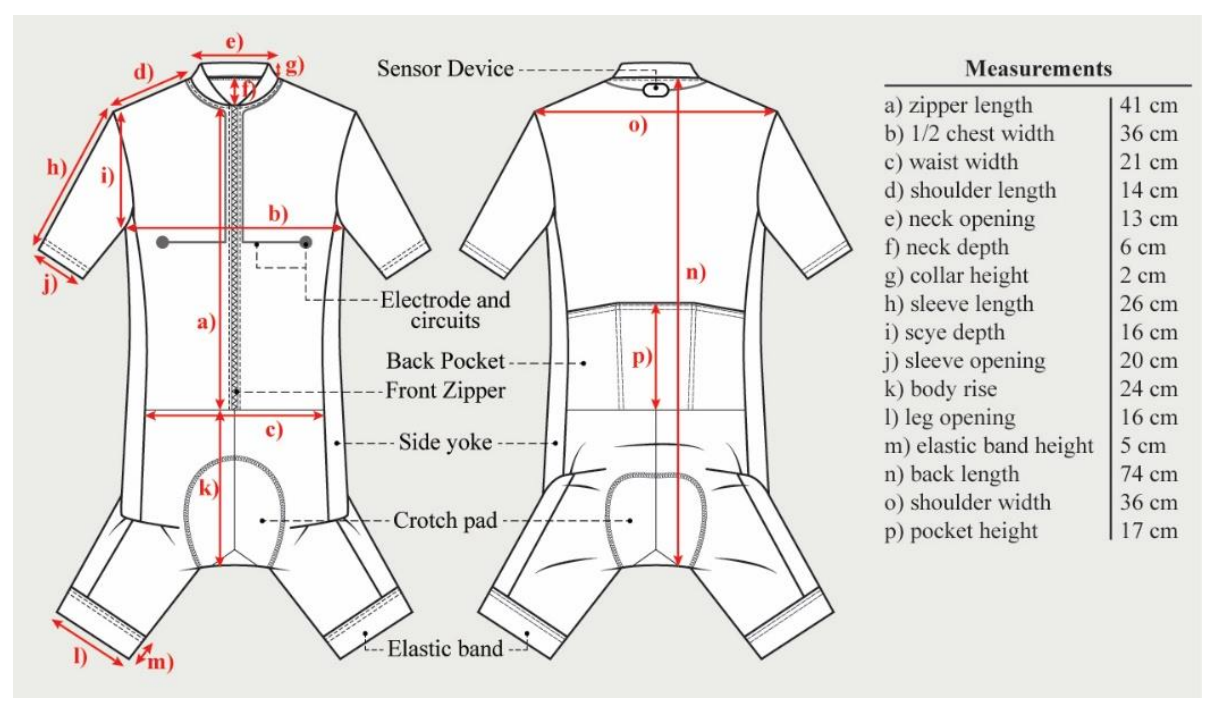

Fig. 1. Detailed drawing of the skinsuit, with specifications for size $S$

\subsection{Choosing the E-textile}

Before building a prototype, several conductive materials - woven fabric (Statex Bremen), knitted fabric (Statex Silverell 1), silver ink (Dupont PE828) and conductive silicone (ELASTOSIL® LR 3162 A/B) - were studied, to check their performance as 
ECG electrodes. For that, elastic bands were produced in the fabric selected for the cycling skinsuit and electrodes were embedded using the appropriate technique for each material (Fig. 2). Thermoplastic polyurethane (TPU) was used on the connections and on the border of the electrodes to isolate the connections from the body and to attach the electrodes to the fabric, maintaining the electrodes visible. Snaps were attached to the end of the connection to plug the monitoring device. The snaps were covered by a piece of fabric, so they do not contact the skin.

In the conductive fabric version (Fig. $2 \mathrm{a}, 2 \mathrm{~b}$ and $2 \mathrm{c}$ ) the fabrics are sewn to the elastic band and in the knitted version (Fig. 2d), the conductive fabric is attached with adhesive. In the electrodes of Fig. 2c, a $3 \mathrm{~mm}$ sponge was put inside the electrode to create some volume. The conductive silicone comprises two components, mixed together in a ratio of 1:1. The mixture was coated onto the knitted fabric (Fig. 2e) and cured at $160^{\circ} \mathrm{C}$, for 10 minutes. For the printed version (Fig. 2f), the ink was applied using a brush and it was then cured at $80^{\circ} \mathrm{C}$ for 20 minutes. The connections were isolated with TPU film, except in the case of the silicone, where this was not needed, since the fabric used to cover the snap also covered the short silicone circuit.

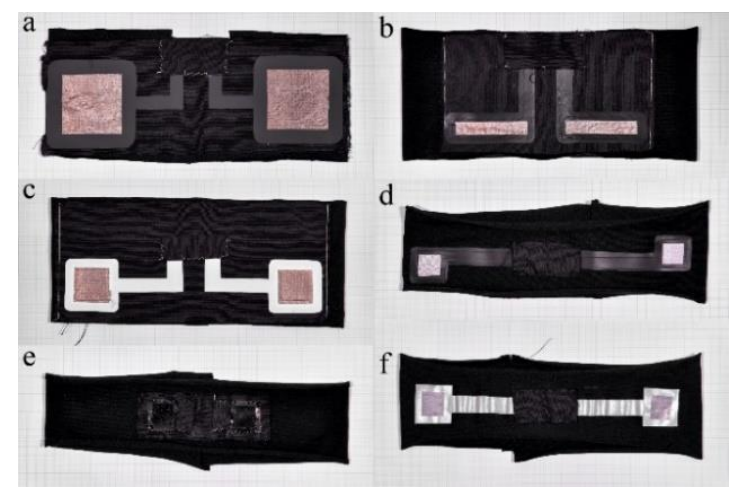

Fig. 2. Stretch bands: a) and b) woven fabric; c) woven fabric with sponge; d) knitted fabric; e) conductive silicone; f) printed with conductive ink

For heart rate measurement, the Polar Beat app was used, which provides real-time information about heart rate and parameters related to cycling (speed, distance...).

\subsection{Prototyping}

According to the design specification, a prototype was prepared for size $\mathrm{S}$ (small). At first, a prototype of the upper garment was built to check for measurements of the torso, which would then define the measurements of the rest of the body. This is an easy way to check if measurements are correct without building the whole garment. The patterns for the bottom were designed and a new prototype was made, comprising the upper and bottom parts, adding the zipper in the front, but keeping aside other details (pockets...). Finally, after measures were checked, a prototype of the whole garment, considering 
all details (except the e-textile) was assembled, in order to understand its construction, but also to check the fitting.

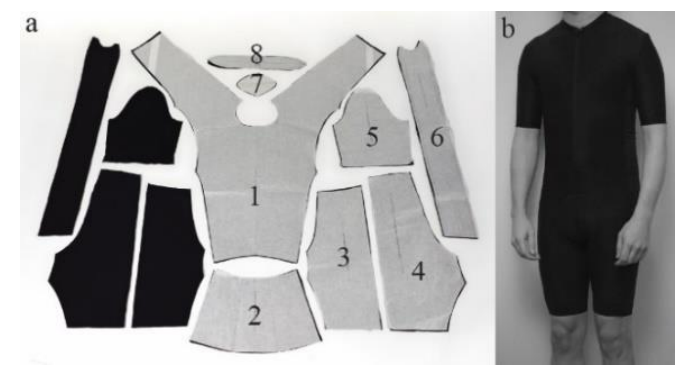

Fig. 3. Garment prototype: a) Patterns cut (1) torso; 2) back pocket; 3) front leg; 4) back leg; 5) sleeve; 6) side yoke; 7) crotch yoke; 8) collar); b) fitting

\section{$4 \quad$ Results}

With all the electrodes, except the conductive silicone based electrodes, the mobile app showed a heart rate, when wetted. With dry electrodes, it was very hard to get a reading. It was only possible to get a reading when applying high pressure on the electrodes. In dry state, the electrodes with the sponge (elastic band $c$ ) gave better readings, because the sponge promotes better contact with the skin, but even in this version the readings were very unstable. It is easier to get a response with wet electrodes, as can be seen from Fig. 4, which shows an example of the heart rate shown in the app, using elastic band $c$, where four stages of exercise can be view: a) static position, b) walking, c) running and d) relaxing.

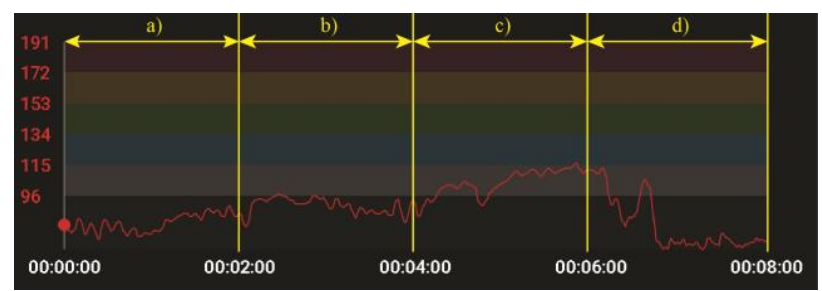

Fig. 4. Heart rate graphic acquired from the app, using the elastic band $c$

The electrodes made with conductive fabric (Bremen) do not have elasticity, unlike its counterparts, the knitted, printed or coated electrodes, which make these last ones more suitable for a garment fitted to the body and, therefore, needing to be stretched.

\section{Discussion}

If a garment with good elasticity is required, and considering that the silicone electrodes don't provide any reading, the best electrodes are the knitted and printed versions. The 
printed method resulted in connections that increase their electrical resistance when deformed and do not recover to the initial value. This may occur because of spaces created in the print after stretching. On the other hand, the electrodes with the sponge provided the best response. The volume of this electrode assembly compresses the electrodes against the skin, therefore, improving the contact with the skin and the reading.

There is a relevant design difference between using conductive ink and fabric. When printing on a thin fabric, the ink is visible on the other side of the fabric, which raises aesthetic concerns. Moreover, given this issue, the connections need to be isolated from both sides. This means that they will be visible and the designer will have to design a pattern that meets the aesthetic requirements. On the other hand, attaching the knitted fabric with a dielectric TPU adhesive will not only hide the circuits inside the garment, but will also isolate it from the outside, making it invisible.

The electrodes produced were tested in stretch bands, which comprise short connections along with the electrode. They may not have the same performance in the suit, given that the connections follow a longer track. A prototype of the suit with embedded electrodes for heart rate monitoring is being prepared for testing, following the above results.

\section{Conclusion}

Developing appealing and functional new products with e-textiles asks for a multidisciplinary approach encompassing design and engineering expertise that changes the way a product is thought and designed.

In this paper, the design process of a cycling smart garment was presented. Several approaches to using e-textiles as electrodes to measure heart rate were reported and a prototype of a cycling suit was made. This kind of work involves expertise in fashion and clothing design, as well as textile and electronics engineering, requiring close cooperation in a team.

The next step will be to produce a prototype of the same suit, but this time with the electrodes embedded. Future work may include exploration of other ways of integrating technology into clothing.

Aknowlegdements. This work is financed by FEDER funds through the Competitivity Factors Operational Programme - COMPETE, by national funds through FCT - Foundation for Science and Technology within the scope of the project POCI-01-0145FEDER-007136 and by TSSiPRO-NORTE-01-0145-FEDER-000015 funded by the regional operational program NORTE 2020, under the PORTUGAL 2020 Partnership.

\section{References}

1. Carvalho, H., Catarino, A., Rocha, A., Postolache, O.: Health Monitoring using Textile Sensors and Electrodes: An overview and integration of technologies. In: IEEE Int. Symp. on Medical Measurements and Appl. (MeMeA), Lisbon (2014) 
2. Paiva, A., Catarino, A., Carvalho, H., Postolache, O., Postolache, G.: Development of Dry Textile Electrodes for Electromiography. In: $9^{\text {th }}$ Int. Conf. on Sensing Technology (ICST), pp. 447-451, Aukland (2015)

3. Pylatiuk, C., Müller-Riederer, M., Kargov, A., Schulz, S., Schill, O., Reischl, M., Bretthauer, G.: Comparison of Surface EMG Monitoring Electrodes for Long-term Use in Rehabilitation Device Control. In: IEEE Int. Conf. on Rehab. Robotics, pp. 300-304, Kyoto (2009)

4. Löfhede, J., Seoane, F., Thordstein, M.: Textile Electrodes for EEG Recording - A Pilot Study. Sensors. 12, 16907-16919 (2012)

5. Puurtinen, M., Komulainen, S., Kauppinen, P., Malmivuo, J., Hyttinen, J.: Measurement of noise and impedance of dry and wet textile electrodes, and textile electrodes with hydrogel. Proceedings of the 28th IEEE Engineering of Medicine and Biology Soc. Annu. Int. Conf. 6012-6015 (2006)

6. Tao, D., Zhang, H., Wu, Z., Li, G.: Real-Time Performance of Textile Electrodes in Electromyogram Pattern-Recognition Based Prosthesis Control. In: Proc. of the IEEE-EMBS Int. Conf. on Biomedical and Health Informatics, pp. 487-490 (2012)

7. De Rossi, D., Rocha, A.M., Abreu, M.J., Fardin, D., Da Silva, J., Ferreira, J., Tavares, V., Correia, M., Dias, R.: E-Legging for Monitoring the Human Locomotion Patterns. J. of Textile Eng. 59:6, 153-158 (2013)

8. Yao, M.: Real-Time Monitoring of Heart Rate using Wellness Belt equipped with Electrocardiagram Sensors. In: Proc. of the $35^{\text {th }}$ Chin. Control Con., Changdu, (2016)

9. Zięba, J., Frydrysiak, M., Blaszczyk, J.: Textronic clothing with resistance textile sensor to monitoring ferquency of human breathing. In: IEEE Int. Symp. on Medical Measurements and Appl. Proc. (MeMeA), Budapest (2012)

10. Manero, R.B.R., Shafti, A., Michael, B., Grewal, J., Fernandez, J.L.R, Althoefer, K.: Wearable Embrodered Muscle Activity Sensing Device for the Human Upper Leg. In: 2016 IEEE $38^{\text {th }}$ Annu. Int. Conf. on the Eng. in Medicine and Biology Soc. (EMBC), pp. 6062-6065. Orlando (2016)

11. Ma, Y.C., Chao, Y.P., Tsai, T.Y.: Smart Clothes - Prototyping of a Health Monitoring Platform. In: IEEE $3^{\text {rd }}$ Int. Conf. on Consumer Electron., Berlin (2013)

12. Frydrysiak, M., Tesiorwski, L.: Wearable Care System for ELderly People. Int. J. of Pharma Medicine and Biological Sci. 5: 3, 171-177 (2016)

13. Carre Technologies Inc.: Getting Started Guide for iPhone and OS X Users. Carre Technologies Inc. (2013)

14. G. Krishnamurthy. (2013). AiQ's BioMan Biomonitoring Shirt and Other Smart Clothing Technology. https://www.medgadget.com. Accessed Jan. 6, 2018.

15. Berger. J. (2015). The Most Interesting Wearable in Fitness: With Athos, Has the Personal Trainer Met its Match?. http://www.gearpatrol.com. Accessed Jan. 6, 2018

16. Pesola, A.: Reduced muscle Activity, sedentary time and cardio-metabolic benefits: effectiveness of a one-year family-based cluster randomized controlled trial. Ph.D. Thesis, Faculty of Sport and Health Sci., University of Jyväskylä, Jyväskylä (2016)

17. Petri. (2016). MBody Allsport 6 Channel. http://www.myontec.com. Accessed on Jan. 6, 2018. 